\title{
LA DISFUNCIONALIDAD FAMILIAR Y LOS NIVELES DE DEPRESIÓN EN ADOLESCENTES DE UNA UNIDAD EDUCATIVA RURAL EN AMBATO, ECUADOR.
}

\author{
FAMILY DISFUNCTIONALITY AND LEVELS OF DEPRESSION IN \\ ADOLESCENTS OF A RURAL EDUCATIONAL UNIT IN AMBATO, \\ ECUADOR
}

Recebimento: 06/08/2017- Aceite: 12/11/2017- Publicação: 23/12/2017

Processo de Avaliação: Double Blind Review

Luis Marcelo Mantilla-Falcón ${ }^{1}$

Doctor en Ciencias de la Educación, Magíster en Docencia Universitaria y

Administración Educativa.

Docente de la Universidad Técnica de Ambato

luismmantilla@uta.edu.ec

Cristina Alomaliza

Psicóloga de la Carrera de Psicología Clínica de la Universidad Técnica de Ambato.

Cristina.alomaliza19@hotmail.com

\section{RESUMEN}

La población mundial, dadas las condiciones actuales de la sociedad, tiene mayores problemas en cuanto a sus factores psicológicos, emocionales y de orden fisiológico, uno de ellos que más recurrencia presenta es la depresión, la misma que es generada por diferentes causas, pero en este estudio se busca la correlación entre la depresión y la disfuncionalidad familiar en adolescentes de una Unidad Educativa Rural de la ciudad de Ambato, Ecuador. La investigación es descriptiva, transversal, no experimental, empírica, la misma que se realizó a 60 estudiantes comprendidos en edades de 15 a 18 años y de los dos géneros. Los resultados demuestran una correlación $r$ de Pearson de 0,694 y la prueba de Ji cuadrado es altamente significativa con un $p$-value de 0,0000

PALABRAS CLAVE: Depresión, Disfuncionalidad familiar, Test de Beck, Test FF-SIL, familia.

\section{ABSTRACT}

The world's population, given the current conditions of society, has the biggest problems in terms of their psychological, emotional and physiological order factors, one more recurrence presents is depression, the same as is generated by different causes, but in

\footnotetext{
${ }^{1}$ Autor para correspondência: Universidad Técnica de Ambato- UTA- Av. de los Chasquis, Ambato, Ecuador
} 
this study the correlation between depression and family dysfunction in adolescents in a Rural Education Unit of the Ambato city, Ecuador. The research is descriptive, transversal and not experimental, empirical, the same as 60 students ranging in age from 15 to 18 and of both genders was performed. The results show a correlation $r 0.694$ and Pearson chi-square test is highly significant with a p-value of 0.0000.

KEYWORDS: depression, family dysfunction, Beck Test, Test FF-SIL, family.

\section{INTRODUCCIÓN}

Hablar de familia, es referirse al núcleo germinador de la sociedad con todas sus manifestaciones e implicaciones, lo que hace la familia, hace la sociedad, por ello es que "la funcionalidad familiar juega un papel importante en el desarrollo social y psicológico de los miembros que constituyen el núcleo familiar" (Cogollo, Gómez, Arcos, Ruíz, \& Campo-Arias, 2009). En la misma línea de pensamiento, algunos autores señalan que la funcionalidad familiar se describe como la capacidad para enfrentar y superar cada una de las etapas del ciclo vital y las crisis por las que atraviesa (Hernández-Castillo, CargillFoster, \& Gutiérrez-Hernández, 2011).

La incidencia de la funcionalidad (o disfuncionalidad) familiar se ve asociada con muchos factores, como por ejemplo, los intentos de suicidios de sus miembros como lo referencia Gaona y otros (2012) al exponer que "la funcionalidad familiar es una variable que puede influir con el intento de suicidio debido a que dependiendo de cómo funcione el hogar de las personas, estas tienden a comportarse del mismo modo, recurriendo al intento de suicidio cuando se encuentra afectada la funcionalidad familiar".

El abordaje del funcionamiento familiar se encuentra en la literatura desde diferentes categorías y perspectivas, por ejemplo para Satir (1986), los modelos de comunicación son prioritarios en su criterio; otros autores como Walsh (1982), determinan la funcionabilidad familiar a partir de la forma en que la familia resuelve sus problemas o como Epstein, Baldwin y Bishop (1983), que plantean tres tareas: básicas (satisfacción de las necesidades materiales de la familia), de desarrollo (ciclo vital) y arriesgadas (capacidad de la familia para resolver las crisis), y consideran familia funcional a aquella que aborda con eficacia las tres (Pérez, de la Cuesta, Louro, \& Bayarre, 1997).

Chagoya (1985) expresa que la funcionabilidad familiar viene dada por la manera en que ésta enfrenta las crisis, valora la forma en que se permiten las expresiones de afecto, así como el crecimiento individual de sus miembros; se produce la interacción entre ellos sobre la base del respeto a la autonomía y el espacio del otro.

En la actualidad para muchos autores la funcionalidad familiar es considerada como "la capacidad para enfrentar y superar cada una de las etapas del ciclo vital y las crisis por las que atraviesa. Una familia funcional es aquella capaz de cumplir con las tareas encomendadas, de acuerdo a un ciclo vital en que se encuentre y en relación con las demandas que percibe del medio ambiente externo (Hernández-Castillo, CargillFoster, \& Gutiérrez-Hernández, 2011).

En temas de depresión en adolescentes, los estudios tienen una larga historia, los datos son evidentes, recogidos por Morla, Saad y Saad (2006) señalan que en la población 
general se dan cifras de $0,3 \%$ en la edad preescolar (Kashani \& Sherman, 1988), 1,8\% de depresión mayor y $6,4 \%$ de trastorno distímico en escolares (Polaina-Lorente \& Doménech, 1988) y 4,9\% en adolescentes de 14 a 16 años (Kashani \& Sherman, 1988), por lo que se puede observar una tasa de depresión con aumento progresivo en función de la edad.

La literatura académica (Melipillán, Cova, Rincón, \& Valdivia, 2008) recoge que la depresión en adolescentes es un problema significativo, asociado a considerable impedimento funcional $\mathrm{y}$ a un mayor riesgo de trastornos depresivos en edades posteriores de la vida (Harrington, 2002). Aproximadamente, un 2 a 5\% de los adolescentes reúne los criterios de trastorno depresivo mayor en un corte transversal, y alrededor de un $16 \%$ han experimentado un trastorno depresivo alguna vez a la edad de 16 años (Costello \& Angold, 2016).

Muchos son los indicadores que evidencian la presencia de sintomatología depresiva como bien lo explica Nardi (2004) En la adolescencia la depresión del tono del humor se presenta con aspectos extremadamente variables: a veces con expresiones comportamentales particularmente impactantes (crisis de llanto, desesperación, rabia), otras veces con actitudes marcadamente inhibidas (cierre relacional, mutismo, retiro de las inversiones emocionales); en otros casos, puede estar "enmascarada" por trastornos psicosomáticos o por una serie de conductas riesgosas (tóxico dependencia, búsqueda del peligro como una forma de desafío divino al destino).

El problema de la depresión es preocupante, en consecuencia, es importante destacar que la Organización Mundial de la Salud, OMS, (2003), ha establecido que la depresión es el trastorno mental más frecuente y discapacitante actualmente en el mundo, y que además afecta por lo menos al $5 \%$ de la población mundial: Iniciada por acontecimientos vitales adversos o problemáticos, como la pérdida de familiares, ruptura sentimental, desempleo, post parto, entre otras (...) el $25 \%$ de la población lo padece al menos una vez en su vida (Ferrel, Vélez, \& Ferrel, 2014).

La fenomenología de la depresión tiene connotaciones importantes en la vida del ser humano, las implicaciones son diversas y se manifiestan de diferentes formas como bien lo detalla García (2009) para quien "La depresión se trata de un trastorno psíquico, habitualmente recurrente, que causa una alteración del estado de ánimo de tipo depresivo (tristeza) a menudo acompañado de ansiedad, en el que pueden darse, además, otros síntomas psíquicos de inhibición, sensación de vacío y desinterés general, disminución de la comunicación y del contacto social, alteraciones del apetito y el sueño (insomnio tardío) agitación o enlentecimiento psicomotor, sentimientos de culpa y de incapacidad, ideas de muerte e incluso intento de suicidio, etc.".

La misma OMS (2016) afirma que "La depresión es un trastorno mental frecuente y una de las principales causas de discapacidad en todo el mundo. Se calcula que afecta a más de 350 millones de personas en todo el mundo, con mayor prevalencia en las mujeres que en los hombres".

Corroborando a la información de la OMS, García (2009) evidencia algunas estadísticas: Depresión mayor del 10 al 15\% en mujeres y del 5 al $12 \%$ en hombres; 
Distimia simple, 3\% de la población; Trastorno Bipolar I entre 0,4 y 1\%; Trastorno bipolar II el 0,5\%; Trastorno ciclotímico del $0,4 \%$ al $1 \%$ de la población.

La literatura científica evidencia que los síntomas más recurrentes en lo adolescentes con problemas de depresión son: aumento o descenso de peso, hipo o hipersomnia, disminución del rendimiento académico, problemas de atención y dificultad para la concentración, anergia, ausencia de motivación, baja autoestima, evitación de actividades lúdicas, disminución en la interacción social, entre otras (García, 2009).

Esta alteración psicológica se vuelve, con el tiempo en un problema de salud pública mundial. Al revisar algunos datos este es el panorama: Actualmente es la cuarta enfermedad discapacitante, pero podría llegar al segundo en el 2020, se presenta entre el 5 y el 8\% de la población. En Europa 1 de cada 5 personas padecerá depresión a lo largo de su vida; En EEUU, alrededor del 10\% de las mujeres y el 4\% de los hombres han tomado algún antidepresivo en su vida; El riesgo de sufrir un trastorno depresivo en su vida está entre el 6 y 10\% en hombres y el 12 al 20\% en mujeres (Zarragoitía-Alonso, 2011).

Básicamente este trastorno es muy complejo, recurrente y con serias implicaciones en la salud psicológica y fisiológica del ser humano.

\section{METODOLOGÍA}

La presente es una investigación cualicuantitativa de carácter descriptivo, no experimental y transversal. Los elementos de observación fueron los estudiantes de octavo 20 (33\%), noveno 21 (35\%) y décimo 19 (32\%) año de Educación General Básica, (Básica Superior, sistema ecuatoriano), de una Unidad Educativa del sector rural de la ciudad de Ambato, Ecuador. Participaron 60 estudiantes comprendidos en edades de 15 a 18 años y de los dos géneros: masculino 37 (62\%) femenino 23 (38\%), con muestreo no probabilístico de orden criterial. Se les aplicó el test de Beck para la determinación de la depresión juvenil y la prueba de percepción del funcionamiento familiar (FF-SIL). El trabajo de campo se efectuó in-situ en la jornada académica de clases, previo el consentimiento de los progenitores. El alfa de Cronbach para el test de Beck es de 0,907 y para el FF-SIL es de $0,979$.

El test de Beck con sus 21 ítems, fue aplicado y analizado en función de sus escalas y baremos como sigue: Depresión ausente o mínima: 0-9 puntos; Depresión leve: 10-16 puntos; Depresión moderada: 17-29 puntos; Depresión grave: más de 30 puntos (Ministerio de Sanidad, Servicios Sociales e Igualdad, 2014). Del mismo modo el test de funcionamiento familiar FF-SIL se ajustó a: Funcional: 70-57 puntos; Moderadamente funcional: 56-43 puntos; Disfuncional: 42-28 puntos; Severamente disfuncional: 27-14 puntos (Pérez, de la Cuesta, Louro, \& Bayarre, 1997).

\section{RESULTADOS}

Las dos variables en análisis en este conjunto de estudiantes se evidencian en la tabla 1 donde se consolida la información más representativa. 
Tabla 1. Disfuncionalidad familiar y depresión

\begin{tabular}{lcclcc}
\hline & DEPRESIÓN & \multicolumn{3}{c}{ DISFUNCIONALIDAD } \\
\hline NIVELES & Frecuencia & Porcentaje & & Frecuencia & Porcentaje \\
Extrema & 16 & 26,7 & Disfuncional & 16 & 26,7 \\
Intermitente & 3 & 5,0 & Funcional & 2 & 3,3 \\
Leve & 9 & 15,0 & Moderada & 25 & 41,7 \\
Moderada & 12 & 20,0 & Severa & 17 & 28,3 \\
Normal & 20 & 33,3 & Total & 60 & 100,0 \\
Total & 60 & 100,0 & & &
\end{tabular}

Fuente: encuesta

La información presenta fuerte presencia de casos de depresión extrema con el $27 \%$ aproximadamente, que también se relaciona con los altos niveles de disfuncionalidad familiar severa que igualmente alcanza un porcentaje del $28,3 \%$.

Muchos estudios giran alrededor de la prevalencia de depresión en adolescentes, pero muy pocos estudios informan de asociación a funcionalidad familiar (LeyvaJiménez, Hernández-Juarez, Nava-Jiménez, \& López-Gaona, 2007). La prevalencia de disfuncionalidad familiar en familias con hijos adolescentes puede encontrarse entre el $22 \%$ y el 33\% (Zapata-Gallardo, y otros, 2007). La prevalencia de síntomas depresivos con importancia clínica en adolescentes se encuentra alrededor del $40 \%$ al 50\% (Cogollo \& Campo-Arias, 2007). Sin embargo, pocos estudios muestran la relación entre funcionalidad familiar y síntomas depresivos con importancia clínica; los pocos estudios disponibles informan que la disfuncionalidad familiar incrementa el riesgo de síntomas depresivos con importancia clínica (McArdle, y otros, 2002).

En un análisis de asociación de las dos variables en estudio mediante tabla de contingencias, se determina que los hogares con disfuncionalidad severa, presentan los índices mayores de depresión juvenil. (ver tabla No. 2.). 
Tabla 2. Asociación de variables: Depresión y disfuncionalidad familiar.

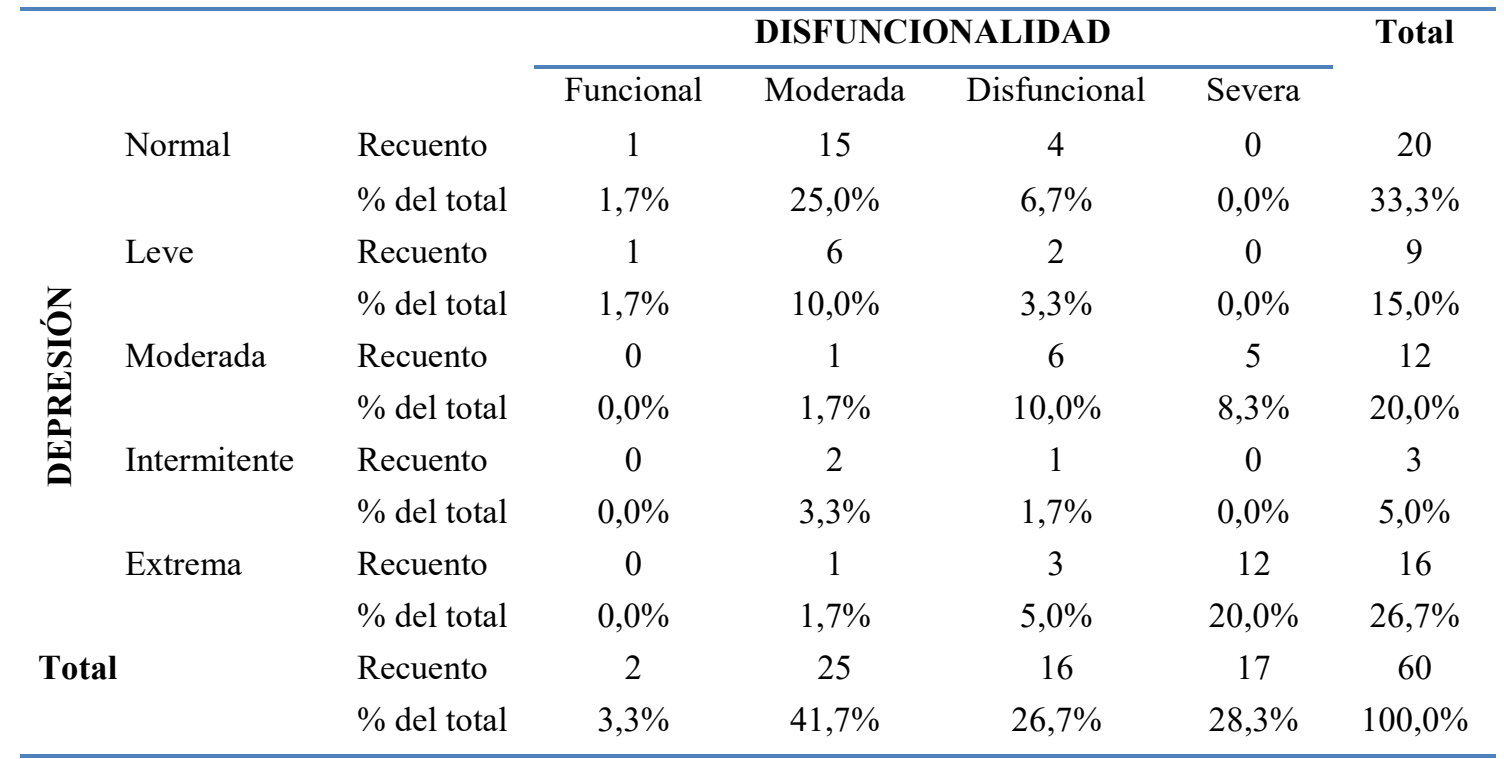

Fuente: Encuesta, 2017.

La información es clara y se evidencia que el 28,3\% de la población estudiantil, proviene de hogares con disfuncionalidad, asimismo se verifica que el $20 \%$ presentan depresión severa. Mediante análisis de Ji cuadrado se determina una asociación altamente significativa con un p-value de 0,0000 y X2 de 43,3 con 12 grados de libertad. La correlación (r) de Pearson para estos datos se encuentra en 0,694 con p-value de 0,0000.

El análisis de la información de campo demuestra que de los 16 sujetos que presentan depresión extrema, el $81 \%$ son los de menor edad (15 años) y se encuentran en octavo año de educación, por el contrario, de los 20 estudiantes con calificación de "normal" el 60\% están en noveno año de Educación General Básica.

En cuanto a la disfuncionalidad familiar los datos evidencian que en octavo año el $65 \%$ de los 20 estudiantes participantes provienen de hogares con disfuncionalidad severa y solo dos son los estudiantes que demuestran abiertamente provenir de un hogar "funcional" representando el 3,3\%.

Si se analiza la información entre edad y depresión, los siguientes son los datos más significativos: los estudiantes de 15 años, alcanza el 21,7\% de depresión extrema, le sigue en orden de importancia los estudiantes de 16 años con el 3,3\%, sin embargo, el $22 \%$ aproximadamente de los estudiantes de 16 años, tienen depresión en los rangos de normal y leve. Para los estudiantes de 18 años, su mayor porcentaje está en depresión moderada con el 16,7\%. Técnicamente, los estudiantes de 17 años no tienen problemas. Del mismo modo, la prueba de Ji cuadrado presenta una asociación significativa con pvalue de 0,0000 y X2 de 63,3 con 12 grados de libertad.

Los datos empíricos en cuanto a género y depresión determinan que los hombres tienen el $15 \%$ de depresión extrema y moderada; las mujeres el $11,7 \%$ de extrema y el $13,3 \%$ de "normal". No hay diferencia estadística significativa entre género y depresión.

La disfuncionalidad familiar y edades se aprecia en la figura 1. 
Fig. 1. Edad y disfuncionalidad familiar en términos porcentuales

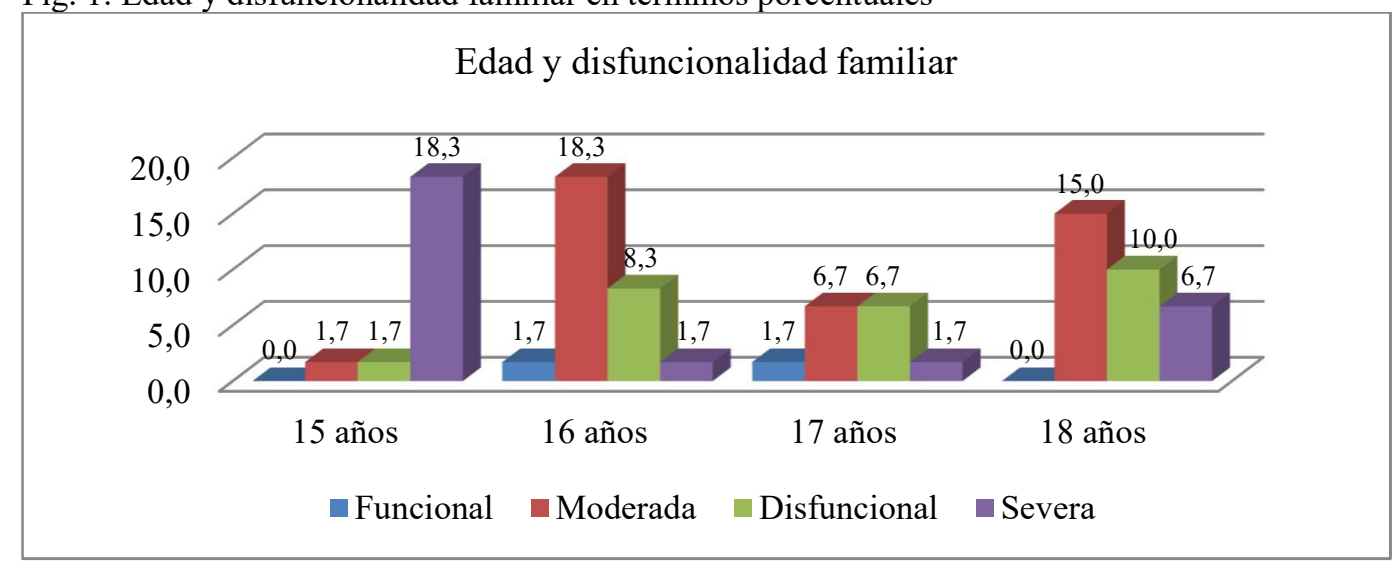

Elaborado por investigadores, 2017.

La fenomenología de la disfuncionalidad en los hogares tiene importantes connotaciones y eso se evidencia que la categoría de "severa" sea la más recurrente y con mayores porcentajes, no menos importante es la categoría de "moderada".

\section{CONCLUSIONES}

Existe un grado de dependencia muy significativo entre la depresión juvenil y la disfuncionalidad de los hogares de proveniencia de los estudiantes demostrando, de este modo, una asociación o correlación muy ajustada entre ellas.

Los estudiantes de menor edad son los que más propensos están a sufrir de factores depresivos en cualquiera de los niveles de la escala de Beck, ellos son muy perceptivos a los acontecimientos del hogar y están más expuestos a sufrir afectación en su salud psicológica.

Se evidencia una fuerte disfuncionalidad familiar en los hogares estudiados que tienen directa implicación con los niveles de depresión que presentan los estudiantes de los tres cursos, y estos niveles disminuyen conforme avanza la edad. La disfuncionalidad es fuerte en este segmento poblacional de estudio, primero por ser de una zona rural de la ciudad con niveles económicos bajos y muy bajos.

La depresión en los adolescentes se presenta en un momento de grandes cambios personales, cuando hombres y mujeres están definiendo una identidad distinta a la de sus padres, lidiando con asuntos de género y su sexualidad emergente, y tomando decisiones por primera vez en sus vidas (NIMH, 2009).

Queda como recomendación a los actores educativos y profesionales de la salud emocional profundizar en el estudio y diseñar planes de atención primaria para este segmento poblacional para mejorar su calidad de vida y bienestar.

\section{BIBLIOGRAFÍA}

Chagoya, L. (1985). La dinámica familiar y patología. En D. Gutiérrez, La familia, medio propiciador o inhibidor del desarrollo humano. México D.F.: Prensa Médica. 
Cogollo, Z., \& Campo-Arias, A. (abril-junio de 2007). Asociación entre síntomas depresivos con importancia clínica y rendimiento académico en estudiantes de Cartagena, Colombia. Ciencias de la Salud, 33-39. Obtenido de http://www.scielo.org.co/pdf/recis/v5n1/v5n1a4.pdf

Cogollo, Z., Gómez, E., Arcos, O., Ruíz, I., \& Campo-Arias, A. (2009). Asociación entre disfuncionalidad familiar y síntomas depresivos con importancia clínica en estudiantes de Cartagena, Colombia. Revista Colombiana de Psiquiatría, 38(4), 637-644. Obtenido de http://www.scielo.org.co/pdf/rcp/v38n4/v38n4a05.pdf

Costello, E. J., \& Angold, A. (2016). Developmental epidemiology. En D. Cichetti, \& D. Cohen (Edits.), Developmental Psychopathology (Tercera ed., págs. 41-75). Ney Jersey: John Wiley and Sons Inc.

Epstein, N., Baldwin, L., \& Bishop, D. (1983). The McMasteer family assessment device. Journal of Marital and Family Therapy, 9(2), 171-180. Obtenido de https://www.ntnu.no/c/document_library/get_file?uuid=cd377890-a31d-4692-a9b8$47 \mathrm{c} 563844862$ \& groupId $=10293$

Ferrel, F. R., Vélez, J., \& Ferrel, L. F. (2014). Factores psicológicos en adolescentes escolarizados con bajo rendimiento académico: depresión y autoestima. Encuentros, 12(2), 35-47. Obtenido de http://www.scielo.org.co/pdf/encu/v12n2/v12n2a03.pdf

Gaona, J. B., Bohórquez, A., Gómez, P. A., Ramírez, Y. D., Barrientos, G., Peñaloza, L. N., \& Guecha, E. A. (Diciembre de 2012). Incidencia de los antecedentes de funcionalidad familiar, consumo de sustancias psicoactivas y enfermedad mental frente al intento de sucicidio. Ciencia y Cuidado, 9(2). Obtenido de https://dialnet.unirioja.es/descarga/articulo/4114532.pdf

García, A. (Marzo de 2009). La depresión en adolescentes. Estudios de Juventud(84), 85105. Obtenido de http://www.injuve.es/sites/default/files/RJ84-07.pdf

Harrington, R. C. (2002). Affective disorders. En M. Rutter, \& E. Taylor (Edits.), Child and adolescent psychiatry (págs. 463-485). Oxford: Blackwell Publishing Science.

Hernández-Castillo, L., Cargill-Foster, N. R., \& Gutiérrez-Hernández, G. (2011). Funcionalidad familiar y conducta de riesgo en estudiantes de nivel medio superior Jonuta, Tabasco 2011. Salud en Tabasco, 18(1), 14-24. Obtenido de http://www.redalyc.org/pdf/487/48724427004.pdf

Kashani, J. H., \& Sherman, D. D. (1988). Chilhood depression: epidemiology, etiological model, and treatment implications. Integr Psychiatry, 6, 1-8.

Leyva-Jiménez, R., Hernández-Juarez, G., Nava-Jiménez, G., \& López-Gaona, V. (2007). Depresión en adolescentes y funcionamiento familiar. Rev Med Inst Mex Seguro Soc, 45(3), 225-232. Obtenido de http://www.medigraphic.com/pdfs/imss/im2007/im073d.pdf 
McArdle, P., Wiegersma, A., Gilvarry, E., Kolte, B., McCarthy, S., Fitzgerald, M., ... Quensel, S. (2002). European adolescent substance use: the roles of family structure function and gender. Addiction(97), 329-336. doi:10.1046/j.1360-0443.2002.00066.x

Melipillán, R., Cova, F., Rincón, P., \& Valdivia, M. (2008). Propiedades Psicométricas del Inventario de Depresión de Beck-II en Adolescentes Chilenos. Terapia Psicológica, 26(1), 59-69. Obtenido de http://www.redalyc.org/articulo.oa?id=78526105

Ministerio de Sanidad, Servicios Sociales e Igualdad. (2014). Guía de Práctica Clínica sobre el Manejo de la Depresión en el Adulto. Madrid, España: Ministerio de Sanidad, Servicios Sociales e Igualdad. Obtenido de http://www.guiasalud.es/GPC/GPC_534_Depresion_Adulto_Avaliat_compl.pdf

Morla, R., Saad, E., \& Saad, J. (abril-junio de 2006). Depresión en adolescentes y desestructuración familiar en la ciudad de Guayaquil, Ecuador. Revista Colombiana de Psiquiatría, $\quad X X X V(2), \quad 149-166 . \quad$ Obtenido de http://www.redalyc.org/articulo.oa?id=80635203

Nardi, B. (2004). La depresión adolescente. Psicoperspectivas, III(1), 95-126. Obtenido de http://www.psicoperspectivas.cl/index.php/psicoperspectivas/article/viewFile/14/14

NIMH. (2009). Depresión. EEUU: Instituto Nacional de la Salud Mental. Obtenido de https://www.nimh.nih.gov/health/publications/espanol/depresion/depresion_38791.pdf

OMS. (2003). El trastorno mental mas frecuente. Diario Córdova.

OMS. (abril de 2016). Trastornos mentales. Obtenido de http://www.who.int/mediacentre/factsheets/fs396/es/

Pérez, E., de la Cuesta, D., Louro, I., \& Bayarre, H. (1997). Funcionamiento familiar. Construcción y validación de un instrumento. Ciencia Ergo Sun, 4(1), 63-66. Obtenido de http://cienciaergosum.uaemex.mx/index.php/ergosum/article/view/4004/3054

Polaina-Lorente, A., \& Doménech, E. (1988). La depresión en niños españoles de cuarto de E.G.B. Barcelona: Geygi.

Satir, V. (1986). Psicoterapia familiar conjunta (Segunda ed.). México D.F.: La Prensa Médica Mexicana S.A. de C.V.

Walsh, F. (1982). Conceptualization and normal family functioning. En F. Walsh (Ed.), Normal family processes (págs. 3-42). New York, New York, USA: The Guildford Press, XXI.

Zapata-Gallardo, J. N., Figueroa-Gutiérrez, M., Méndez-Delgado, N., Miranda-Lozano, V. M., Linares-Segovia, B., Carrada-Bravo, T., . . . Rayas-Lundes, A. M. (septiembreoctubre de 2007). Depresión asociada a la disfunción familiar en la adolescencia. Bol Med Hosp Infant Mex, 64. Obtenido de http://www.medigraphic.com/pdfs/bmhim/hi2007/hi075e.pdf 
Zarragoitía-Alonso, I. (2011). Depresión. Generalidades y particularidades. La Habana: Editorial Ciencias Médicas. ECIMED. Obtenido de http://newpsi.bvspsi.org.br/ebooks2010/pt/Acervo_files/depresion-cuba.pdf 